\title{
Data Fast Transmission Method in Wireless Vehicle Ad-hoc Network
}

\author{
Professor Dr. Sorush Niknamian \\ BSC in Applied Mathematics (IAUCTB), BSC in Mechatronics Engineering (SUT) \\ and $\mathrm{PhD}$ in Cell and Molecular Biology and Military Medicine, Liberty University \\ (LU), USA \\ Email: so.ninamian@gmail.com
}

\begin{abstract}
In vehicular ad hoc networks, the current method does not consider the delay of data transmission, resulting in slower vehicle data transmission speed. A vehicle data transmission method based on backbone network is proposed in this paper. Firstly, the characteristics of vehicle ad hoc network are analyzed. Based on the statistics of the road, the vehicle cluster is composed of the vehicles parking on the roadside and no roadside according to the different directions of the vehicle driving. The backbone network is constructed on the basis of the cluster of vehicles, and the data transmission between the vehicles is implemented by the data transmission method of overlay network. This method can overcome the disadvantages of traditional data transmission methods, improve the efficiency of on-board data transmission, and complete the research on fast data transmission method in wireless vehicle ad hoc network. The experimental results show that the proposed method can achieve higher data transmission success rate with lower data transmission overhead and smaller transmission delay.
\end{abstract}


Keywords: Wireless ad-hoc network, Vehicle data, Fast transmission, Backbone network

\section{Introduction}

Traffic accidents are still a bottleneck restricting the development of society (Tao et al., 2015). Taking intelligent transportation measures can reduce the incidence of traffic accidents, improve road utilization and vehicle travel efficiency, and effectively guarantee people's property and life safety (Afanasiev et al., 2017). The rise of vehicle ad-hoc network has provided a new direction for the development of intelligent transportation, which has attracted the attention of scholars from various countries (Bamiedakis et al., 2015). Vehicle ad-hoc network is mainly through infrastructure between vehicles and roadside communication as the basis, to provide the optimal decision route information to the driver, and guide driving; meanwhile, the traffic information is given for road management, providing data support for road supervision and management department (Chen et al., 2016; Zhang et al., 2016). However, vehicle ad-hoc network has unique technical requirements and constraints, so the vehicle data transmission network is facing greater challenges (Wang, 2015).

In this regard, researchers have put forward some data transmission methods in vehicle ad-hoc network. Gao et al (Gao et al., 2015) proposed a reliable transmission method of vehicle remote data. In the area covered by the network, the $3 \mathrm{G}$ technology was used to transmit the remote data of the vehicle. In the area where the network could not be covered, the communication of the Beidou 
navigation system was used to carry on the transmission of the vehicle data (Bardsiri, 2018; Cayirci and de Oliveira, 2018; Panov and Nagrebetskaya, 2018). The experimental results showed that the method had the reliability of data transmission, but the speed of data transmission was slow. Wang et al (2016) proposed a transmission optimization strategy of vehicle data based on the stopping theory. The stopping problem of data transmission constraints and the effective range of the transmission data are constructed, and the transmission rate of the transmitting terminal of the vehicular radio in the periodic channel is obtained. Experimental results showed that this method had relatively small data transmission energy consumption, and the success rate of data transmission was relatively high, but there was a slow speed of data transmission.

\section{Data fast transmission method of wireless ad-hoc network vehicle}

\subsection{Vehicle routing decision}

In order to transmit the data of the wireless vehicular ad-hoc network quickly, the decision is made on the route selection, and the transmission delay is evaluated at different time periods. ${ }^{d_{i j}}$ is the link that the message passes through, and ${ }^{e_{i j}}$ is the delay generated by data transmission. According to the length of the section $e_{i j}$ , the density and the average speed of the vehicle, the calculation is carried out. It is assumed that the data is sent from the intersection ${ }^{I_{i}}$, and the data transmission delay pass through the section $e_{i j}$ to the destination is $D_{i j} .{ }^{N(j)}$ is The intersection set adjacent to the intersection ${ }^{I_{i}}$. Then, 


$$
D_{i j}=d_{i j}+\sum_{k \in N(j)} P_{j k} D_{j k}
$$

In formula (1), ${ }^{{ }_{j k}}$ is the probability that data is transmitted from intersection $I_{i}$ to intersection $e_{i j}$. Because the discontinuous connection of wireless ad-hoc network, when the data carrier passes through the intersection ${ }^{I_{i}}$, it will encounter the unsuitable vehicle, resulting in the fact of that the data can not be transmitted to the expected direction, so that the actual data transmission direction has probability (Hanawal et al., 2016). As a result, ${ }^{\sum_{k \in N(j)} P_{j k} D_{j k}}$ indicates the delay required for that the data will be transmitted from the intersection $I_{i}$ to the destination. Using the formula (1), the $D_{m m}$ equation corresponding to the road segment $e_{m m}$ in the wireless ad-hoc network is listed. Figure 1 shows the network diagram of the road. If the destination of the data transmission is the intersection $I_{e}$, the corresponding equation is expressed by the formula (2):

$$
\left\{\begin{array}{l}
D_{a b}=d_{a b}+P_{b a} D_{b a}+P_{b c} D_{b c} \\
D_{a c}=d_{a c} \\
D_{b a}=d_{b a}+P_{a b} D_{a b}+P_{a c} D_{a c} \\
D_{b c}=d_{b c} \\
D_{c a}=0 \\
D_{c b}=0
\end{array}\right.
$$

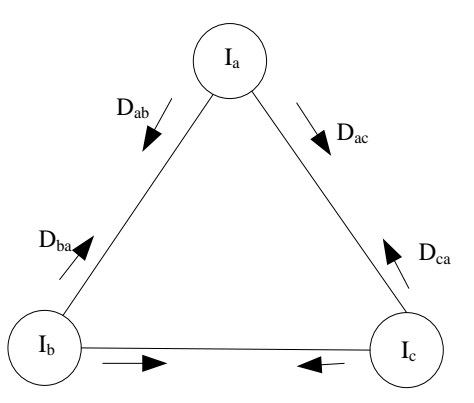




\section{Figure 1. Network diagram of road}

The solution of the equation can be solved, and the corresponding $D_{m m}$ in the outlet section $e_{m m}$ can be obtained. ${ }^{D_{m m}}$ is used to make decisions on road selection.

Because the data cannot be transmitted to the worse vehicle in the current direction, so under the condition of data carrier segment ${ }^{e_{j k c}}$, the probability of data transmission along different roads is:

$$
P_{j k n / j k c}= \begin{cases}P_{j k m}^{\prime} . & \forall m \prec c \\ 1-\sum_{s=1}^{c-1} P_{j k s}^{\prime}, & m=c \\ 0, & \forall m \prec c\end{cases}
$$

By the formula (3), the driving vehicle in the intersection ${ }^{{ }}$is made statistics. The probability in the direction ${ }^{e_{j k c}}$ is ${ }^{Q_{k c}}$, and ${ }^{P_{j k n}}$ can be obtained as follows:

$$
P_{j k n}=\sum_{c=1}^{n} Q_{j k c} P_{j k n / j k c}
$$

\subsection{Design of backbone network of vehicles}

Usually, there are no roadside parking lots on both sides of the road in the city, and the roadside has a large number of parking spaces. The parking vehicles on roadside and no roadside on the same road are formed into a cluster of vehicles. Because the average occupancy rate of parking lot is relatively high, the probability of that the vehicle parking on the roadside can find out nearby parking vehicles in the range of its communication is larger. Therefore, the parking vehicles on the 
roadside can be connected with the rest parking vehicles in the cluster (Nemov et al., 2016).

Figure 2 represents the vehicle's parking cluster. Among them, $H 1$ and $H 2$ are the two cluster heads in the cluster, and $M 1$ and $M 10$ are nodes of the members. The process of building a vehicle cluster is that the roadside node at the end of the road is a cluster head, and when the vehicle enters or leaves, it will meet one of the cluster heads. The cluster head mainly manages the network to deal with the joining and leaving of the vehicle nodes. As ${ }^{Q H 1}$ and ${ }^{Q H 2}$ in Figure 2, the standby cluster head is a vehicle that is parked close to the cluster head. The standby cluster head has a robust effect on the vehicle cluster (Sun et al., 2016).

Data transmission within cluster is following this way: after cluster head is determined, cluster members will transmit their data to cluster heads by broadcasting, and record the node ID passed by data. For nodes that can not communicate with cluster heads, the cluster heads receive ${ }^{n}$ data copies that arrive first when the data is received. According to the sequence of these duplicates reaching the cluster head, the data transmission path of the ${ }^{n}$ data duplicates is marked as the optimal path. After receiving the copy of the ${ }^{n}$ data, the cluster head replies the path information along the optimal path. The above process is illustrated in Figure 3. The node ${ }^{i}$ in the graph is the node of the vehicle cluster member. $H 1$ represents the node of the cluster heads, and the vehicle path $(i, j, k, H 1)$ is the best path from ${ }^{i}$ to cluster head. 


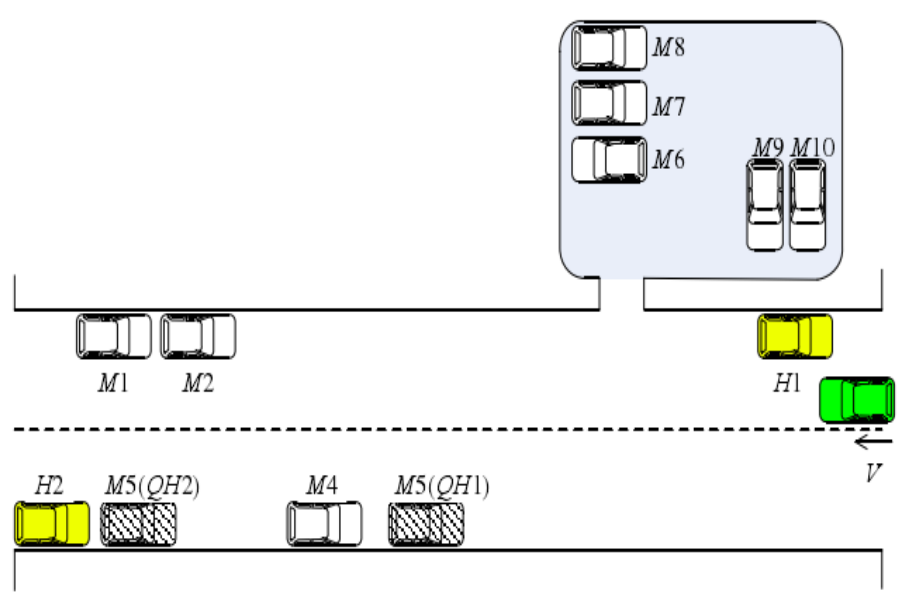

Figure 2. Vehicle cluster

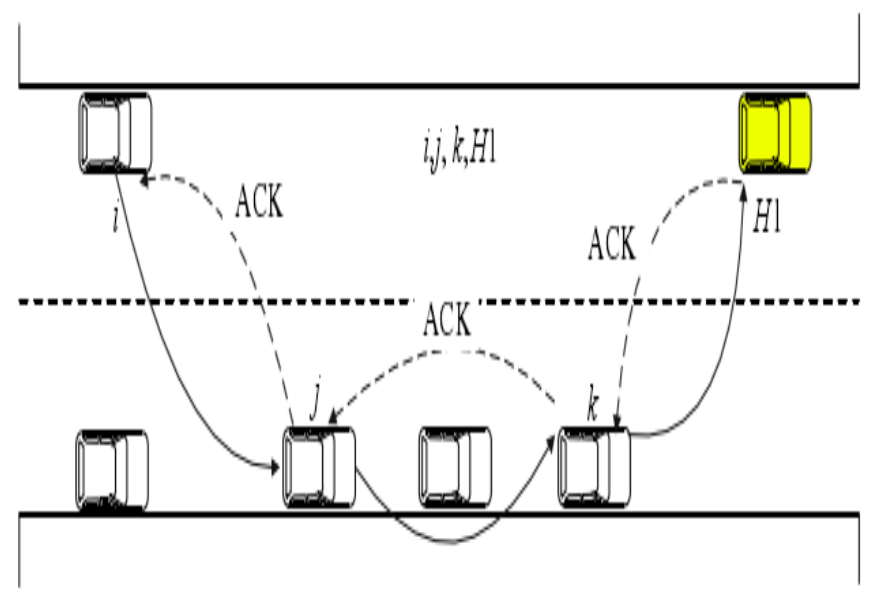

Figure 3. Transmission in cluster

In order to reduce the cost of vehicle data transmission, member nodes periodically report data to cluster heads. When cluster heads transmit data to nodes, the best path is selected firstly. If the optimal path does not exist, sub optimal can be selected. 
In order to realize the fast transmission of the vehicle data in the wireless adhoc network, the virtual parking backbone is constructed on the basis of the vehicle cluster.

(1) Each vehicle cluster initiates the adjacent discovery process. For example, the cluster head nodes of the vehicle cluster $J$ broadcast the data with the cluster head position and $N E i . R E Q$ to the other vehicle clusters. After receiving the $N E i . R E Q$ data, the vehicle cluster $J$ is set as a neighbor cluster. If a vehicle cluster is surrounded by the main road, it is easy to cause the vehicle cluster to stay away from other vehicles, and thus the remaining nodes of the vehicle cluster can not be found.

(2) In order to build all the vehicle clusters into a backbone network, the vehicle cluster and all the neighbourhood vehicle clusters are connected in a virtual connection. Through this process, the network covered by vehicles can form a connected circle $G(V ; E)$, where $V$ is the node of vehicle cluster set, and $E$ is the virtual path set of adjacent vehicles. The structure of the backbone network is shown in Figure 4.

(3) Assuming that members of a backbone network can understand the data and location of other nodes, and then it can be implemented through a simple mechanism. 


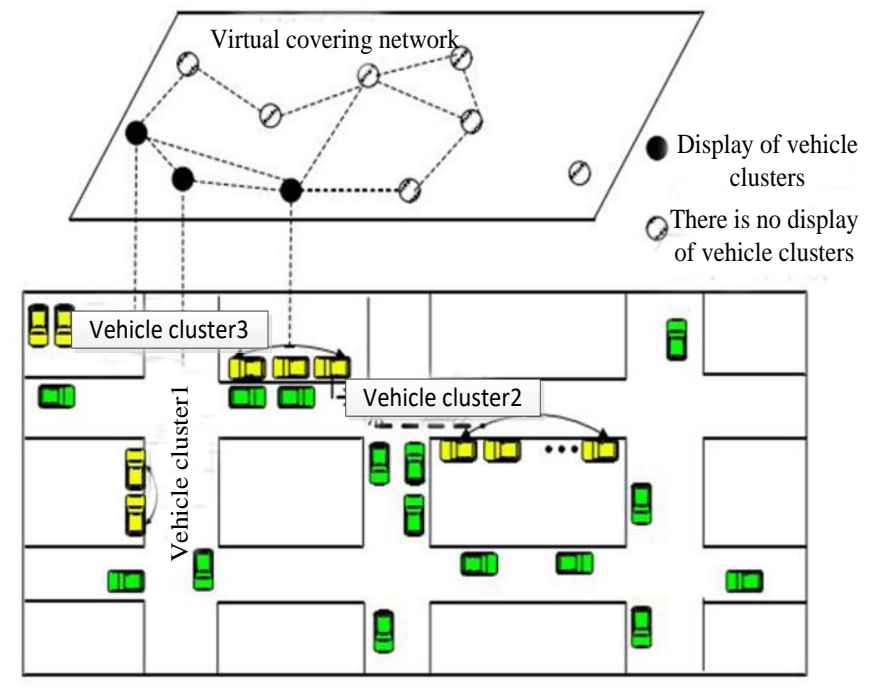

Figure 4. Virtual overlay network

\subsection{The selection of short-distance wireless transmission technology}

In this paper, wireless bridge technology is adopted. Wireless bridge can connect two or more wired local area networks by wireless transmission, and it is the bridge of wireless network. Wireless bridges have two transmission standards, 802.11b and 802.11a. Some specific parameters are shown in Table 1.

Table 1. Transmission standards for wireless bridges

\begin{tabular}{llll}
\hline Standard & Data rate & Frequency band & Transmission distance \\
& & & \\
\hline $802.11 \mathrm{~b}$ & $11 \mathrm{Mbps}$ & $2.4 \mathrm{GHz}$ & Within $30 \mathrm{~km}$ \\
$802.11 \mathrm{a}$ & $54 \mathrm{Mbps}$ & $5.8 \mathrm{GHz}$ & Within $80 \mathrm{~km}$ \\
\hline
\end{tabular}

Wireless bridges are mainly composed of antennas and main devices of wireless bridges. Most wireless bridges provide TCP/IP interfaces to facilitate Ethernet access. Wireless bridges have been widely used in the field of international 
and domestic communications, especially in remote monitoring where it is not convenient to lay communication cables. They can be used for medium and shortdistance high-speed data transmission and long-distance low-rate data transmission. At present, data transmission based on wireless bridge has been applied to many fields, such as campus network coverage construction, industrial park security monitoring, anti-theft, fire prevention and disaster prevention, highway pavement monitoring and so on.

As a widely used wireless network bearing technology, wireless bridge has its unique advantages, which can be summarized as follows: 1. high transmission rate, up to $54 \mathrm{Mbps}$; 2. strong anti-jamming capability; 3. good concealment and confidentiality; 4. strong multi-path interference capability; 5 . high security.

There are three networking modes for wireless bridges.

1. Point-to-point mode: This mode is usually used between two fixed networks. This type of network has long transmission distance, high transmission rate and little influence from outside environment. This paper adopts the point- to- point mode (see as Figure 5).

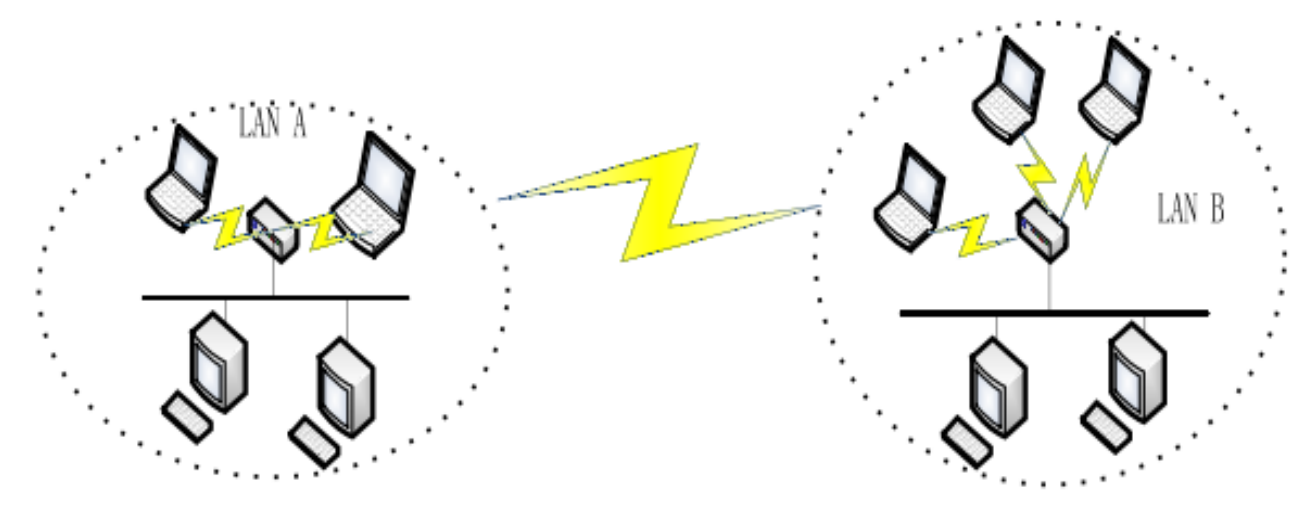




\section{Figure 5. Point-to-point mode}

2. Point-to-multipoint mode: applicable to a central station and multiple remote stations. The advantages of point-to-multipoint mode are as follows: low network cost, simple maintenance and easy debugging. However, this mode also has some shortcomings, which can be seen in the following aspects: firstly, because multiple remote terminals share one central point device, network delay increases, transmission rate decreases, and if the central device is damaged, the whole network will be paralyzed; secondly, the central device uses omnidirectional antenna. When the antenna works, the velocity of the wave will diffuse in all directions, which will cause the transmission power to be greatly attenuated, the transmission rate of the network will be reduced, and the remote station in the farther position may not be able to obtain reliable network connection; thirdly, all bridge settings use the same working frequency, and if one remote station is disturbed, other stations will be replaced. At the same frequency, if the situation is worse and multiple remote stations are disturbed, the frequency replacement will be more frequent and more troublesome (see as Figure 6). 


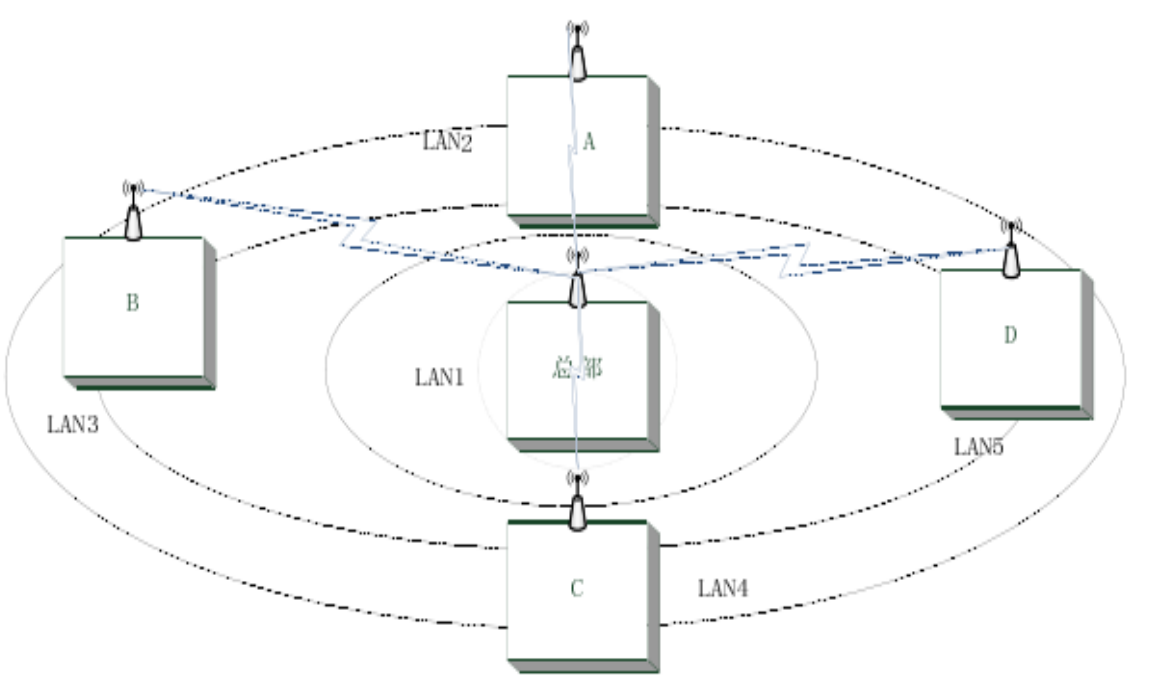

Figure 6. Point-to-multipoint mode

3. Relay station mode: when there are obstacles between two local area networks that need to be connected, wireless relay can be used to bypass obstacles. Because there is no big obstacle between the two locations where the connection is established in this paper, this mode is not used (see as Figure 7).

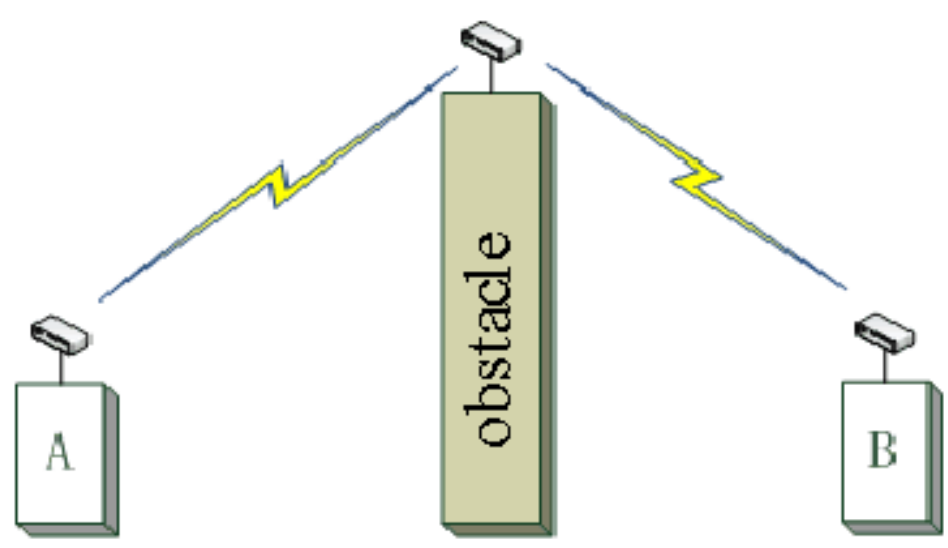

Figure 7. Continuation mode 
According to the above analysis, this paper adopts the point-to-point bridge scheme of $802.11 \mathrm{~b}$ standard.

\subsection{Characteristics of vehicle recording data}

The data recorded by vehicle SIBAS 32 system in wireless ad hoc network include main transformer, converter, main circuit breaker, pantograph, battery, brake control unit, speed sensor, temperature sensor, contactor, relay, cab switch, air conditioner, etc.

When the data is downloaded from the U disk, opening the file and finding that the data is stored in a folder containing multiple. ZIP files. The content of the file is packaged and named by the main CCU (central control unit), the slave CCU, the main TCU and the slave TCU. The main CCU is responsible for the vehicle control of the traction unit. It reads instructions and information from the peripheral and wire train bus (WTB), and sends control signals and feedback information to the peripheral WTB. In addition, the main CCU has the following functions: controlling the main circuit breaker and pantograph; setting traction point of traction control unit (TCU); protecting transformer; controlling vehicle power supply; controlling the front-end connection and front-end movable valve;

Control instructions are set for various control devices, such as doors, air conditioning, lighting, etc.; the diagnosis of safety circuits, fire alarm systems and bogies is monitored; digital and analog inputs and outputs are controlled through decentralized input and output stations; brake control is parked; central control unit 
diagnosis and WTB and MVB communication diagnosis; by connecting the gateway to the train bus (WTB), the configuration of the EMU and the long train set is determined and detected.

Slave CCU is running the same program with the main CCU, it can not control the process actively. The slave CCU can monitor the working state of the main CCU and take over the work of the main CCU whenever the main CCU fails. However, both the main and the CCU have active protection functions for highvoltage hardware devices.

\subsection{Adaptive transmission mechanism of vehicle-borne data based on transmission power control}

Another important factor that affects the data transmission performance of vehicles is the transmission power of vehicle-borne data. In wireless ad hoc networks, it is generally believed that the transmission power and distance of vehicle-borne data are one-to-one correspondence. The use of higher transmission power means that it has a larger transmission distance, and the vehicle-borne data transmission has better robustness. It also has a larger interference range to the vehicle-borne signal of the surrounding vehicles, and the higher the interference intensity of the vehicle-borne signal. On the contrary, lower transmission power means smaller transmission distance, less robustness of vehicle-borne data transmission, and smaller interference range and lower signal interference intensity to surrounding vehicles. If all vehicles use the same data transmission power, the transmission range is the same. However, this obviously does not apply to the 
rapidly changing wireless ad hoc network topology. Traditional power control schemes need to consider energy consumption. In wireless ad hoc networks, for some roadside facility nodes that use renewable energy to supply power, the energy consumption problem needs to be considered, and there is no need to consider the vehicle nodes and the roadside facility nodes with power supply. For vehicle-borne data transmission, it is necessary to consider the appropriate coverage of vehicleborne signals for different applications, as well as the large transmission range and channel load caused by large transmission power. Therefore, it is necessary to control the transmission power of vehicle-borne data adaptively so as to control the interference intensity of vehicle-borne signal and prevent the occurrence of channel congestion under the condition that the channel can be used reasonably to meet the application requirements. Following are several adaptive transmission mechanisms of vehicle-borne data based on transmission power control proposed by researchers.

Power control of vehicle-borne data transmission based on traffic density: the transmission power is adjusted to cover a certain number of vehicles by traffic density, and the traffic density is estimated by traffic flow theory. The corresponding relationship between vehicle speed and traffic density in free and congested traffic is analyzed in detail. Vehicles can estimate the current traffic flow state first, and then estimate the density based on the specific state, which improves the accuracy of the estimation. By using this method, the vehicle can adjust the transmission power without the need of extra information exchange, and the realtime performance is high. However, the dynamic change of topology in vehicular 
ad hoc networks is not taken into account, and the fast switching of different traffic states is not taken into account in the algorithm design, which will affect the reliability of the algorithm.

The method is extended to study the traffic flow models of three kinds of traffic time, including morning, morning peak and evening peak. A large number of real data are used to study the accuracy of traffic density estimation, so as to better carry out adaptive control of vehicle data transmission power.

Power control of vehicle data transmission considering user fairness: in the above study, all vehicles in the default wireless ad hoc network system follow the same adaptive transmission opportunity. This ensures fairness between nodes. The fairness of power control for vehicle data transmission is specially studied. The author believes that it is not enough for a single vehicle to know only the nodes within one hop communication range. Without knowing the environment of neighbor nodes, transmission power control can not guarantee the fairness between users. The essence of the research is to propose a rigorous and fair power control scheme for vehicle data transmission. Therefore, a cooperative communication method between users is proposed to understand the status of nodes in multi-hop range. In this scheme, each vehicle node collects the transmission power information of the neighbor node at the current time and broadcasts the collected information. Through this cooperative mechanism, the nodes can expand the vehicle nodes' understanding of the transmission power of the nodes within and outside the communication range as much as possible. Then, the author proposes a 
transmission power control scheme. By calculating the maximum transmission power threshold under the global power situation, the transmission power of the vehicle itself is increased as much as possible to ensure the reliable acceptance of vehicle-borne data by the neighbor nodes. On the other hand, the maximum quotient of transmission power is constrained in the threshold to ensure that the channel load of the vehicle node itself and its surrounding will not be too large. The simulation results show that this method can guarantee the fairness between nodes skillfully and effectively, and all nodes can send vehicle data reliably. The disadvantage of this algorithm is that it makes too much use of the cooperation between users. As data interaction also consumes channel resources, the effective utilization of channel decreases.

Considering the fairness of users, a random transmission power selection strategy is proposed in the research process. After specifying an average transmission power and its variance, all vehicle nodes randomly adjust the on-board data transmission power based on the same average and variance. The method proves that a good average setting can guarantee the perception of the whole network. The simulation results show that this random selection method can effectively reduce the full-value collision of vehicle data and improve the global fairness.

\subsection{Vehicle data transmission based on backbone network}


Combined with the above, the backbone network is constructed into weighted connect graph $G(V ; E)$, and the weight $D_{i j}$ of $E_{i j}$ represents the delay of data transmission between adjacent node ${ }^{i}$ and ${ }^{j}$. Figure 8 shows the structure of weighted connect graph.

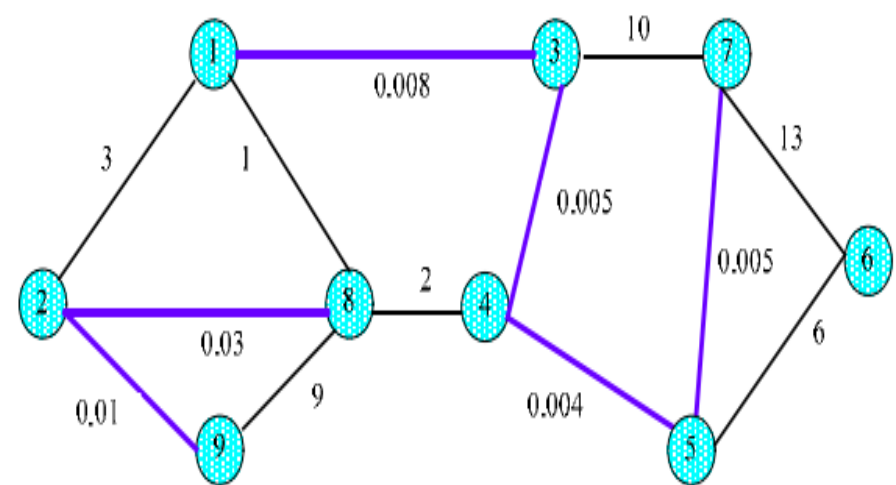

Figure 8. Weighted connect graph

Because the vehicle cluster itself is a road, data transmission on the backbone network can be divided into two parts, which are the transmission of the cluster nodes and the path transmission. Supposing that the data transmission delay of each vehicle cluster node $i$ is $d_{i}$. When the vehicle cluster node $i$ sends data to the vehicle cluster, the data transmission delay from ${ }^{i}$ node to ${ }^{k}$ node is:

$$
T_{i k}=\sum_{i=1}^{m} d_{i}+\sum_{(i, j)=1}^{n} D_{i j}
$$

In formula 5, $m$ indicates the number of nodes that needs to pass through of which the node ${ }^{i}$ passes through the vehicle backbone and transfers the data to the node ${ }^{k} . n$ indicates the number of paths that node ${ }^{i}$ transfers the data needed to 
pass through the vehicle backbone network to node ${ }^{k}$. When the parking density is satisfied, the interior of the vehicle cluster $i$ has connectivity. ${ }^{d}$ is:

$$
d_{i}=\sum_{f=1}^{t} \frac{P_{\text {size }}}{S}
$$

In formula (6), $S$ represents the throughput of the wireless ad-hoc network, $P_{\text {size }}$ represents the size of the data, and ${ }^{t}$ represents the number of jumps that the data transmits within the vehicle cluster $i$. If the suspended vehicle density does not meet the data transmission, it is:

$$
d_{i}=\sum_{i=1}^{t} \frac{P_{\text {size }}}{S}+\frac{l_{i}-t \int_{0}^{R} x \lambda e^{-\lambda x}}{v}
$$

In the formula (7), $l_{i}$ represents the length of the vehicle cluster $i, v$ represents the speed of the vehicle, and the $R$ represents the range of the vehicle for communication. When the vehicle cluster ${ }^{i}$ is connected, ${ }^{d}$ will be very small. When the vehicle cluster does not generate connectivity, it needs to carry the data of vehicles, so $d_{i}$ is relatively large.

$$
D_{i j}^{\prime}=\left\{\begin{array}{l}
D_{i j}, \quad \mu \geq-\frac{1 n(1-\sqrt[m]{1 / 2})}{R} \\
D_{i j}+d_{i}, \mu \prec \frac{1 n(1-\sqrt[m]{1 / 2})}{R}
\end{array}\right.
$$

In formula (8), ${ }^{\mu}$ represents the vehicle density of a ${ }^{i}$ parked in a cluster. After the changes produced by the formula (8), the delay of the data transmission 
generated by the node $i$ to $k$ of the vehicle cluster depends on the $\sum^{\sum_{(i, j)=1}^{n} D_{i j}^{\prime}}$ and requires $\min \left\{\sum_{(i, j)=1}^{n} D_{i j}^{\prime}\right\}$.

According to the statistical results, the parameter $\lambda$ is mostly around 0.5. According to the traffic statistics of each vehicle on different roads and in different time periods, the value of ${ }^{d_{x y}}$ is calculated according to the formula (8).

$$
D_{i j}=\sum_{R_{x y} \in R_{i j}}\left[\frac{l_{x y}\left(1-\lambda^{2} \rho_{x y} l_{x y} \int_{0}^{R} e^{-\lambda x} d x \int_{0}^{R} x e^{-\lambda x} d x\right)}{v_{x y}}+\sum_{i=1}^{\rho_{x x l y}, \rho_{0}^{R x}-2 x x d x} \frac{P_{s i z e}}{S}\right]
$$

After the value of $D_{i j}^{\prime}$ is calculated, the delay matrix of data transmission is constructed by the delay of data transmission between the clusters. According to the delay matrix of data transmission, the shortest path of data transmission delay can be obtained.

$$
M S D=\min \left(\sum_{(i, j) \in E} D_{i j}^{\prime}\right)
$$

\section{Experimental results and analysis}

In this paper, the simulation tool is Network Simulator, and the parameters used in the simulation experiment are expressed in Table 2. In the experiment, the nodes are used to simulate the source nodes to transmit the data, the trajectory of the vehicle nodes is generated by Vanet MobiSim, and the simulation time is 500 S. 


\begin{tabular}{cc}
\hline Parameters & Value \\
\hline Mobile generator & Vanet MobiSim \\
Experimental area $/ \mathrm{m} 2$ & $3000 \times 150$ \\
Range of data transmission R/m & $200-250$ \\
The rate of data transmission / (Mbit·s-1) & 6 \\
Vehicle moving speed / (m-s-1) & $22.2-33.4$ \\
Number of vehicle nodes & $30,60,120,150$ \\
Experimental time /s & 500 \\
Beacon frequency $/ \mathrm{Hz}$ & 1 \\
\hline
\end{tabular}

The ratio of the successful transmission of vehicle data at the intersection is

tested. As shown in Figure 9, the average rate of source and target traffic are ${ }^{\lambda_{1}}$ and $\lambda_{2}$. The process of data arrival is Poisson process. The traveling vehicle will carry the corresponding data packet, transmit it in the intersection area, record the received data packets at the exit $\mathrm{D}$ of the road, and calculate the proportion of the transmission data to the original data.

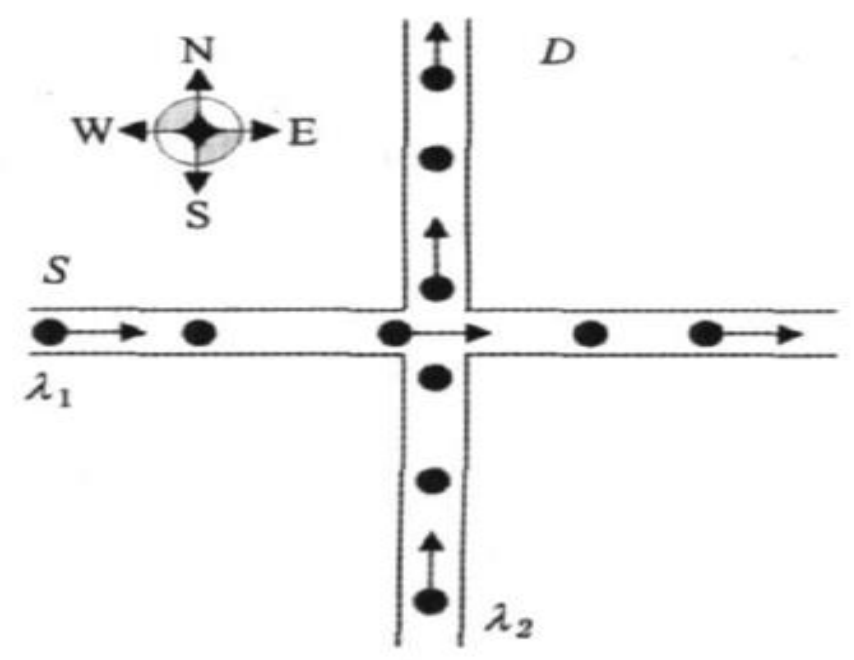

Figure 9. Model of intersection 
By changing the data of vehicle carrying and the arrival rate of vehicles in different directions, we can get the relationship between data single hop transmission and data volume under different road conditions (Figure 10).

The single hop performance of the vehicle's data transmission method based on backbone network proposed in this paper is compared with that of based on mobile gateway. The average arrival rates of vehicle are 0.1 and 0.5 , and two cases of sparse and dense traffic flow are simulated. The effect of data transmission on a mobile gateway depends on the rate ${ }^{\lambda_{2}}$ of arrival of the recipient vehicle. As can be seen from Figure 10, in the case of dense traffic, the ratio of data transmission by the proposed method is kept at a higher level.

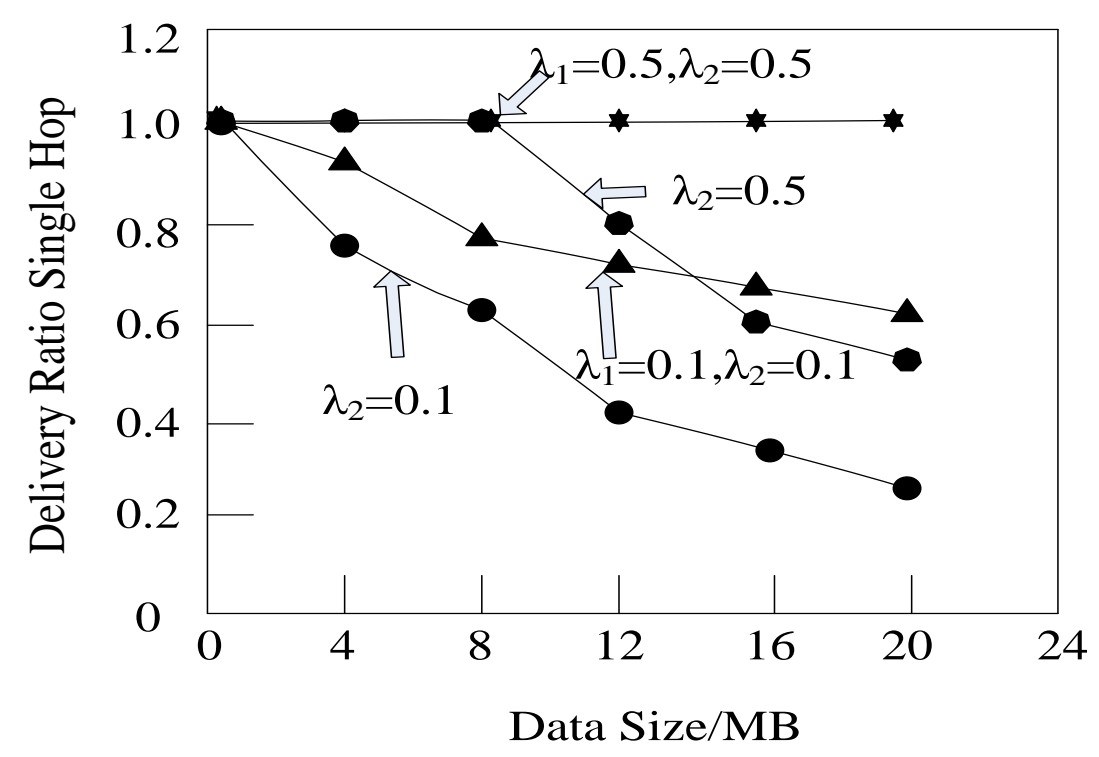

Figure 10. The relationship between the rate of transmission success and the amount of data in a single jump 
To verify the effect of data transmission delay on vehicle data transmission, the modes of data generation and transmission are reset. Figure 11 shows a delayed cumulative distribution map of vehicle data transmission. From Figure 11, it can be seen, compared with the data transmission based on mobile gateway, the vehicle data transmission based on backbone network has little transmission delay, about $90 \%$ of the data can be transferred within 120 s; therefore, using this method, the data of the wireless vehicle ad-hoc network can be transmitted quickly.

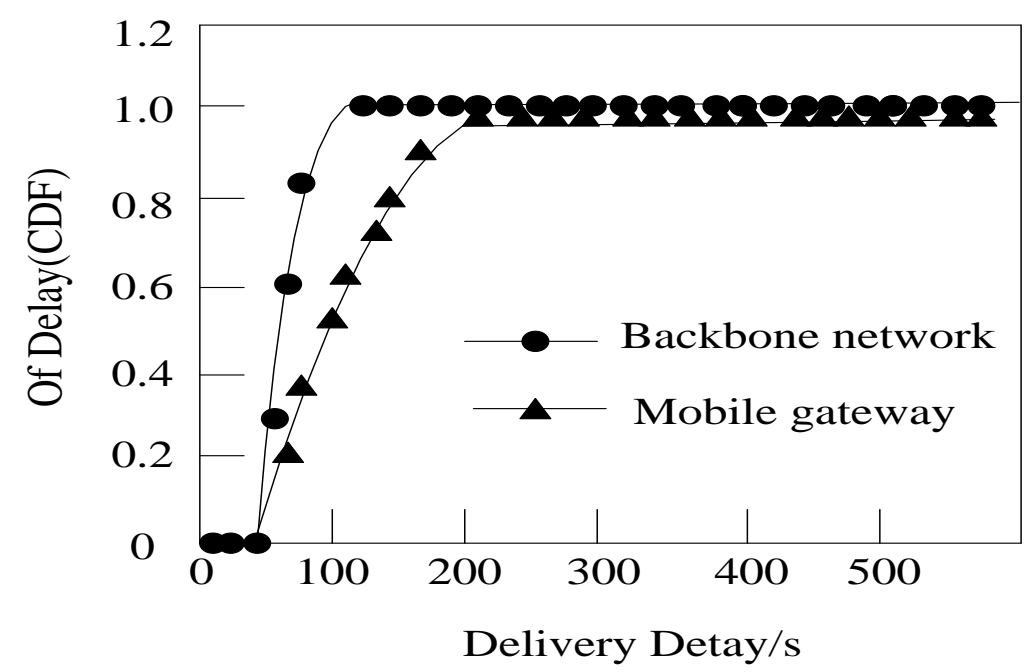

Figure 11. Accumulation distribution of data transmission delay in different methods

\section{Conclusions}

Data transmission is the basis of vehicular ad hoc network application. Vehicular ad hoc networks are data centric wireless networks. This paper proposes a fast transmission method for on-board data based on backbone network. 
Experiments show that this method can achieve higher data fast transmission with smaller transmission cost and delay.

Future research:

(1) According to the actual survey data, the distribution of parked vehicles is improved.

(2) The routing of data under unknown conditions is implemented.

(3) The waste of resources and data redundancy to data transmission caused by parking vehicles needs to reduce.

\section{References}

Afanasiev, M. V., Pratt, R. G., Kamei, R., et al. (2017). Waveform-based simulated annealing of crosshole transmission data: a semi-global method for estimating seismic anisotropy. Geophysical Journal International, 199(3), 1586-1607. DOI: 10.1093/gji/ggu307.

Bamiedakis, N., Chen, J., Westbergh, P., et al. (2015). 40 Gb/s Data Transmission Over a 1-m-Long Multimode Polymer Spiral Waveguide for Board-Level Optical Interconnects. Journal of Lightwave Technology, 33(4), 882-888. DOI: 10.1109/JLT.2014.2371491.

Bardsiri, A.K. (2018). A new combinatorial framework for software services development effort estimation. International Journal of Computers and Applications, 40(1): 14-24. 
Cayirci, E. and de Oliveira, A.S. (2018). Modelling trust and risk for cloud services. Journal of Cloud Computing, 7(1).

Chen, J., Bamiedakis, N., Vasil'Ev, P. P., et al. (2016). High-Bandwidth and Large Coupling Tolerance Graded-Index Multimode Polymer Waveguides for OnBoard High-Speed Optical Interconnects. Journal of Lightwave Technology, 34(12), 2934-2940.DOI: 10.1109/JLT.2015.2500611.

Du L, Gong S, Wang L, et al. (2016). Information-traffic coupled cell transmission model for information spreading dynamics over vehicular ad hoc network on road segments. Transportation Research Part C, 73:30-48.

Feng J, Lu G, Wang H, et al. (2016). Supporting secure spectrum sensing data transmission against SSDH attack in cognitive radio ad hoc networks. Journal of Network \& Computer Applications, 72(C):140-149.

Gao, X.J., Che, M.,Li, H. (2015). Point-to-point 3G Remote Data Transmission Under Heterogeneous Network Standard. Computer Engineering, 41(9), 120-125.

Ghahremani S, Ghanbari M. (2016). Error resilient video transmission in ad hoc networks using layered and multiple description coding. Multimedia Tools \& Applications, 76(6):1-17.

Hanawal, M., Abdelrahman, M., Krunz, M. (2016). Joint Adaptation of Frequency Hopping and Transmission Rate for Anti-jamming Wireless Systems. IEEE Transactions on Mobile Computing, 15(9), 2247-2259. DOI:ieeecomputersociety.org/10.1109/TMC. 2015. 2492556. 
Nemov, S. A., Blagikh, N. M., Allakhkhakh, A. A., et al.(2016). Energy spectrum of holes in $\mathrm{Sb} 2 \mathrm{Te} 2.9 \mathrm{Se}$ 0.1, solid solution according to the data on the transfer phenomena. Physics of the Solid State, 58(11), 2290-2293.

Panov, V.G. and Nagrebetskaya, J.V. (2018). Classification of combined action of binary factors and coxeter groups. Journal of Discrete Mathematical Sciences and Cryptography, 21(3): 661-677.

Peng, Y., Wang, G.C., Huang, S. Q. (2016). An Energy Consumption Optimization Strategy for Data Transmission Based on Optimal Stopping Theory in Mobile Networks. Chinese Journal of Computers, 39(6), 1162-1175.

Ramakrishnan B, Selvi M, Nishanth R B, et al. (2017). An Emergency Message Broadcasting Technique Using Transmission Power Based Clustering Algorithm for Vehicular Ad Hoc Network. Wireless Personal Communications, 94(4):31973216.

Sun, Y., Yan, P. X., Wang, Z. H., et al. (2016). The Parallel Transmission of Power and Data With the Shared Channel for an Inductive Power Transfer System. IEEE Transactions on Power Electronics, 31(8), 5495-5502. DOI: 10.1109/TPEL.2015.2497739.

Tan H, Choi D, Kim P, et al. (2017). Comments on "Dual Authentication and Key Management Techniques for Secure Data Transmission in Vehicular Ad Hoc Networks". IEEE Transactions on Intelligent Transportation Systems, PP (99):13. 
Tao, X., Bodington, D., Reinig, M., et al. (2015). High-speed scanning interferometric focusing by fast measurement of binary transmission matrix for channel demixing. Optics Express, 23(11), 14168-87. DOI: 10.1364/OE.23.014168.

Vijayakumar P, Azees M, Kannan A, et al. (2016). Dual Authentication and Key Management Techniques for Secure Data Transmission in Vehicular Ad Hoc Networks. IEEE Transactions on Intelligent Transportation Systems, 17(4):10151028.

Wang J, Wang J, Zhang X, et al. (2016). Highly reliable MAC protocol optimisation design for data transmission in industrial wireless ad-hoc network. International Journal of Sensor Networks, 21(2):89-97.

Wang Y, Liao T, Wang C. (2016). An Anti-Eavesdrop Transmission Scheduling Scheme Based on Maximizing Secrecy Outage Probability in Ad Hoc Networks. China Communications, 13(1):176-184.

Wang, A.X., Li, Q., Wang, C. S. (2015). Research and Simulation of Data Equilibrium Strategy under High Database Loading. Computer Simulation, 33(3), 327-330.

Zaimi I, Houssaini Z S, Boushaba A, et al. (2016). An improved GPSR protocol to enhance the video quality transmission over vehicular ad hoc networks International Conference on Wireless Networks and Mobile Communications. IEEE, 146-153. 
Zhang, W., Hao, M., Xu, Z. (2016). Communication optimization for RDMA-based science data transmission tools. Journal of Supercomputing, 72(9), 3312-3327. DOI: 10.1007/s11227-015-1399-7. 